\title{
DIAGONALIZATION OF A BOSONIC QUADRATIC FORM USING CCM: APPLICATION ON A SYSTEM WITH TWO INTERPENETRATING SQUARE LATTICE ANTIFERROMAGNETS
}

\author{
S.E. KRÜGER, D. Schmalfuss and J. RIChter \\ Institut für Theoretische Physik, Universität Magdeburg \\ PF 4120, 39016 Magdeburg, Germany
}

\begin{abstract}
While the diagonalization of a quadratic bosonic form can always be done using a Bogolyubov transformation, the practical implementation for systems with a large number of different bosons is a tedious analytical task. Here we use the coupled cluster method to exactly diagonalise such complicated quadratic forms. This yields to a straightforward algorithm which can easily be implemented using computer algebra even for a large number of different bosons. We apply this method on a Heisenberg system with two interpenetrating square lattice antiferromagnets, which is a model for the quasi-2D antiferromagnet $\mathrm{Ba}_{2} \mathrm{Cu}_{3} \mathrm{O}_{4} \mathrm{Cl}_{2}$. Using a four-magnon spin wave approximation we get a complicated Hamiltonian with four different bosons, which is treated with coupled cluster method. Results are presented for magnetic ground state correlations.
\end{abstract}

PACS numbers: 75.50.Ee, 75.30.Ds, 31.15.Dv, 42.50.Lc

\section{Introduction - the model}

It is always possible to diagonalize quadratic bosonic forms (which appear frequently in physics) using a Bogolyubov transformation [1], but it is a tedious analytical task to find one for a complicated form with many different magnons. Therefore we want to show here how the coupled cluster method (CCM), one of the most powerful and universal techniques in quantum many-body theory (see [2] and references therein), can be used in a straightforward scheme to find the exact ground state of such a form.

To be concrete we consider the 2D spin- $1 / 2$ Heisenberg model

$$
H=J_{A A} \sum_{\left\langle i \in A_{1}, j \in A_{2}\right)} S_{i} \boldsymbol{S}_{j}+J_{B B} \sum_{\left\langle i \in B_{1}, j \in B_{2}\right\rangle} S_{i} \boldsymbol{S}_{j}+J_{A B} \sum_{\langle i \in A, j \in B\rangle} S_{i} \boldsymbol{S}_{j},
$$

which is related to the situation in $\mathrm{Ba}_{2} \mathrm{Cu}_{3} \mathrm{O}_{4} \mathrm{Cl}_{2}[3,4]$, a layered quantum antiferromagnet showing significant differences to its parent cuprates like $\mathrm{La}_{2} \mathrm{CuO}_{4}$ (see e.g. [5] for recent experiments). In contrast to $\mathrm{La}_{2} \mathrm{CuO}_{4}$ we have two different types of $\mathrm{Cu}$-sites in the $\mathrm{Cu}-\mathrm{O}$ planes, namely there are additional $\mathrm{Cu}(B)$ atoms located in the centre of every second $\mathrm{Cu}(A)-\mathrm{O}_{2}$ square. Within the $\mathrm{Cu}(A)$ subsystem we have a strong $180^{\circ} \mathrm{Cu}-\mathrm{O}-\mathrm{Cu}$ superexchange yielding to strong antiferromagnetic 
couplings $\left(J_{A A}\right)$ between $\mathrm{Cu}(A)$ atoms, whereas the couplings within the $\mathrm{Cu}(B)$ subsystem $\left(J_{B B}\right)$ and between the subsystems $\left(J_{A B}\right)$ are weaker. A recent calculation of $J_{A A}, J_{B B}$ [6], finding $J_{A A} \approx 10 J_{B B}$ (both antiferromagnetic) agrees with the experimental values [5]. There are also some arguments [6] for a ferromagnetic $\left|J_{A B}\right| \approx J_{B B}$.

In the classical ground state (1) shows for $\left|J_{A B}\right| \leq 2 \sqrt{J_{A A} J_{B B}}$ a Néel-like order for the two subsystems $A$ and $B$, where the energy is degenerated with respect to the angle $\varphi$ between the spins of these two subsystems.

\section{The method}

In this paper we study the ground state properties of (1), using a four-magnon linear spin wave approximation [7] around the classical ground state, i.e. for each of the four sublattices $A_{1}, A_{2}, B_{1}, B_{2}$ of the two coupled bipartite antiferromagnetic square lattices we introduce different bosonic operators. Thus we get for (1)

$$
\begin{aligned}
H= & -\frac{2 N}{3} s^{2}\left(2 J_{A A}+J_{B B}\right)+\sum_{\boldsymbol{k}} H_{k}, \quad \text { with } \\
H_{k}= & 4 J_{A A} s\left[a_{1 k}^{+} a_{1 k}+a_{2 k}^{+} a_{2 k}-\gamma_{\boldsymbol{k} A A}\left(a_{1 k}^{+} a_{2-k}^{+}+a_{1 k} a_{2-k}\right)\right] \\
& +2 J_{B B} s\left[b_{1 k}^{+} b_{1 k}+b_{2 k}^{+} b_{2 k}-\gamma_{\boldsymbol{k} B B}\left(b_{1 k}^{+} b_{2-k}^{+}+b_{1 k} b_{2-k}\right)\right] \\
& +J_{A B} s \frac{1}{2}(1+\cos \varphi)\left(b_{1 k}^{+} a_{1 k}+b_{1 k} a_{1 k}^{+}-b_{2 k}^{+} a_{1-k}^{+}-b_{2 k} a_{1-k}\right) \gamma_{\boldsymbol{k} A B}^{1} \\
& +J_{A B} s \frac{1}{2}(1-\cos \varphi)\left(b_{2 k}^{+} a_{2 k}+b_{2 k} a_{2 k}^{+}-b_{1 k}^{+} a_{2-k}^{+}-b_{1 k} a_{2-k}\right) \gamma_{\boldsymbol{k} A B}^{2} \\
& +J_{A B} s \frac{1}{2}(1-\cos \varphi)\left(b_{2 k}^{+} a_{1 k}+b_{2 k} a_{1 k}^{+}-b_{1 k}^{+} a_{1-k}^{+}-b_{1 k} a_{1-k}\right) \gamma_{\boldsymbol{k} A B}^{1} \\
& +J_{A B} s \frac{1}{2}(1+\cos \varphi)\left(b_{1 k}^{+} a_{2 k}+b_{1 k} a_{2 k}^{+}-b_{2 k}^{+} a_{2-k}^{+}-b_{2 k} a_{2-k}\right) \gamma_{\boldsymbol{k} A B}^{2},
\end{aligned}
$$

using the lattice structure factors $\gamma_{\boldsymbol{k} A A}=\cos \left(k_{x} / 2\right) \cos \left(k_{y} / 2\right), \gamma_{\boldsymbol{k} B B}=\left(\cos k_{x}+\right.$ $\left.\cos k_{y}\right) / 2$ and $\gamma_{k A B}^{1(2)}=\cos \left(k_{x(y)} / 2\right)$.

As stated, we use the coupled cluster method (CCM) to find the exact ground state of (2). To do this we notice the following property of $H$ :

$$
\sum_{\boldsymbol{k}} H_{\boldsymbol{k}}=\sum_{\boldsymbol{k}}\left(H_{k}+H_{-k}\right) / 2 \equiv \sum_{\boldsymbol{k}} H_{k}^{\prime} ; \quad \Rightarrow\left[H_{k}^{\prime}, H_{k^{\prime}}^{\prime}\right]_{-}=0 \quad \forall k, k^{\prime} .
$$

Hence it is possible to treat each $H_{k}^{\prime}$ separately within the CCM, since they all commute with each other. Therefore we have to deal with a bosonic system with eight different bosonic operators $a_{1 \pm k}, a_{2 \pm k}, b_{1 \pm k}, b_{2 \pm k}$ denoted with $a_{1}, \ldots, a_{8}$.

The ket and bra ground state of such a system (i.e a many-mode bosonic field theory with bosonic operators $a_{i}, a_{i}^{+}$in the Hamiltonian) in CCM-SUB $l$ ( $\mathrm{SUB} l$ means products of $l$ or fewer bosonic creation operators) approximation is given by $[2,8]$

$$
\begin{aligned}
& |\Psi\rangle=\mathrm{e}^{S}|0\rangle, \quad S=\sum_{i_{1}, i_{2}, \ldots, i_{l}} A_{i_{1}, i_{2}, \ldots, i_{l}} a_{i_{1}}^{+} a_{i_{2}}^{+} \ldots a_{i_{l}}^{+}, \\
& \langle\tilde{\Psi}|=\langle 0| \tilde{S} \mathrm{e}^{-S}, \quad \tilde{S}=1+\sum_{i_{1}, i_{2}, \ldots, i_{l}} \tilde{A}_{i_{1}, i_{2}, \ldots, i_{l}} a_{i_{1}} a_{i_{2}} \ldots a_{i_{l}},
\end{aligned}
$$


where $|0\rangle$ is the bosonic vacuum state (i.e. $a_{i}|0\rangle=0$ ), and $A_{i_{1} \ldots}$ and $\tilde{A}_{i_{1}} \ldots$ are the CCM correlation coefficients. These coefficients are calculated by two systems of equations (one of them is a system of nonlinear equations)

$$
\frac{\partial \bar{H}}{\partial \tilde{A}_{i_{1} \cdots i_{l}}}=0, \quad \frac{\partial \bar{H}}{\partial A_{i_{1} \cdots i_{l}}}=0, \quad \bar{H}=\langle\tilde{\Psi}|H| \Psi\rangle,
$$

using the expectation value $(\bar{H})$ of the Hamiltonian, i.e. the ground state energy.

Note that the CCM-SUB2 approximation (i.e. having only quadratic terms of bosonic operators in $S$ and $\tilde{S}(5)$ ) gives the exact ground state of a quadratic bosonic Hamiltonian, since the ground state wave function of such a Hamiltonian has the form $|\Psi\rangle=\exp \left(\sum_{i j} f_{i j} a_{i}^{+} a_{j}^{+}\right)|0\rangle$, which can easily be shown using a Bogolyubov transformation (see Appendix). Therefore the CCM correlation operator $S$ (and $\tilde{S}$ respectively) (5) consists of products of two bosonic creation operators only, all other coefficients $A_{i_{1}, \cdots, i_{l}}$ are zero; so we just have to use SUB2.

To calculate the CCM equations (6) easily using computer algebra, we make use of the Bargmann representation $[9,8]$

$$
a^{+} \Leftrightarrow z, \quad a \Leftrightarrow \frac{\mathrm{d}}{\mathrm{d} z}, \quad|0\rangle \Leftrightarrow 1,\left.\quad\left\langle 0\left|f\left(a, a^{+}\right)\right| 0\right\rangle \Leftrightarrow f\left(\frac{\mathrm{d}}{\mathrm{d} z}, z\right)\right|_{z=0},
$$

which maps the original many-mode bosonic field theory into the corresponding (classical) field theory of complex functions in a particular normed space. Therefore instead of bosonic operators we just have to handle with (complex) numbers and differential operators, which is much easier. Once the (partial nonlinear) equations are obtained they can be solved numerically.

\section{Results and conclusions}

We apply the CCM-scheme described above to calculate the exact ground state of (3) and by doing this we get a spin wave approximate ground state of the model (1). We discuss the energy as a function of the angle between spins
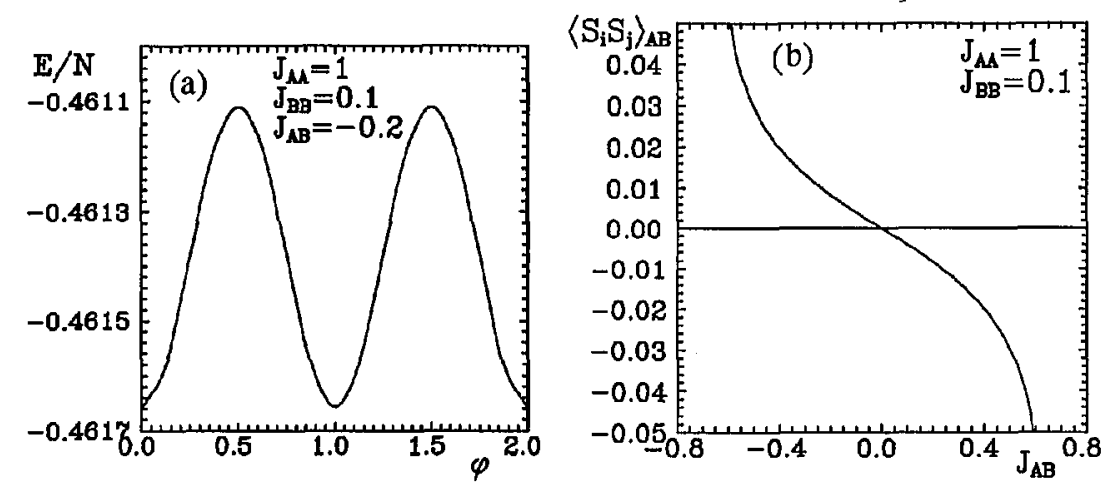

Fig. 1. Ground state of the model (1): (a) energy as a function of the (classical) angle $\varphi$ (in units of $\pi$ ) between the two subsystems $A$ and $B$ (note that in the classical case the energy does not depend on $\varphi$ ); and (b) correlation between spins of these two subsystems in dependence on $J_{A B}$. 
of the two subsystems $A$ and $B$ and the correlation between spins of different subsystems as a function of $J_{A B}$ (Fig. 1). We find as a typical order from disorder effect that the degeneracy of the ground state with respect to the angle $\varphi$ is lifted by quantum fluctuations and a collinear ordering $(\varphi=0, \pi)$ is stabilized. This can clearly be seen by the energy vs. $\varphi$ picture in Fig. 1 and by the correlation $\left\langle S_{i} S_{j}\right\rangle_{A, B}$ vs. $J_{A B}$, which is zero in the classical case, independent of the value of $J_{A B}$ (for $\left|J_{A B}\right| \leq 2 \sqrt{J_{A A} J_{B B}}$ ). In the quantum case however that correlation does depend on $J_{A B}$, showing again an order effect induced by quantum fluctuations.

In addition we find a lowering of the magnetic order within the subsystems $A$ and particular $B$ by frustrating $J_{A B}$ in the quantum case.

\section{Acknowledgments}

This work has been supported by the DFG (project No. Ri 615/7-1).

\section{Appendix}

\section{Proof that CCM-SUB2 gives exact ground state}

Using the fact that a Bogolyubov transformation $\beta_{\nu}=\sum_{\mu}\left(u_{\mu \nu}^{*} a_{\mu}-v_{\mu \nu}^{*} a_{\mu}^{+}\right)$ exactly diagonalizes a quadratic bosonic Hamiltonian with the bosonic operators $a_{i}, a_{i}^{+}$, one can easily show that its ground state must have the form $|\Psi\rangle=$ $\exp \left(\sum_{i j} f_{i j} a_{i}^{+} a_{j}^{+}\right)|0\rangle$, by showing that $\beta_{\nu}|\Psi\rangle=0 \forall \nu$. We use the Bargmann representation (7) and get

$$
\begin{aligned}
& \beta_{\nu}|\Psi\rangle \stackrel{!}{=} 0 \Leftrightarrow \sum_{\mu}\left(u_{\mu \nu}^{*} \frac{\mathrm{d}}{\mathrm{d} z_{\mu}}-v_{\mu \nu}^{*} z_{\mu}\right) \exp \left(\sum_{i j} f_{i j} z_{i} z_{j}\right) \stackrel{!}{=} 0 \quad \forall z_{i} \\
& \Rightarrow \sum_{\mu}\left(u_{\mu \nu}^{*} 2 \sum_{i} f_{i \mu} z_{i}-v_{\mu \nu}^{*} z_{\mu}\right) \stackrel{!}{=} 0 \quad \forall z_{i}, \quad \Rightarrow 2 \sum_{\mu} f_{i \mu} u_{\mu \nu}^{*} \stackrel{!}{=} v_{i \nu}^{*}
\end{aligned}
$$

and this last matrix equation is always fulfilled for some $f_{i \mu}$.

\section{References}

[1] N. Bogolyubov, J. Phys. (USSR) 11, 23 (1947); J.H.P. Colpa, Physica A 93, 327 (1978).

[2] R.F. Bishop, Theor. Chim. Acta 80, 95 (1991).

[3] J. Richter, N.B. Ivanov, R. Hayn, J. Schulenburg, J. Magn. Magn. Mater. 177-181, 737 (1998).

[4] J. Richter, D. Schmalfuß, S. Krüger, Physica B 259-261, 911 (1999).

[5] F.C. Chou, A. Aharony, R.J. Birgeneau, O. Entin-Wohlman, M. Greven, A.B. Harris, M.A. Kastner, Y.J. Kim, D.S. Kleinberg, Y.S. Lee, Q. Zhu, Phys. Rev. Lett. 78, 535 (1997).

[6] H. Rosner, Phys. Rev. B 57, 13660 (1998).

[7] T. Holstein, H. Primakoff, Phys. Rev. 58, 1908 (1940).

[8] J.S. Arponen, R.F. Bishop, Theor. Chim. Acta 80, 289 (1991).

[9] V. Bargmann, Comm. Pure Appl. Math. 14, 180, 187 (1961); ibid. 20, 1 (1967); V. Bargmann, P. Butera, L. Girardello, J.R. Klauder, Rep. Math. Phys. 2, 221 (1971). 